\title{
Strange Attractors in Writing Competence Development from the Perspective of Complexity Theory Based on Sensor Data
}

\author{
Chao Zhang $\mathbb{D}$ \\ College of Chinese Language and Literature, Qufu Normal University, Qufu, China \\ Correspondence should be addressed to Chao Zhang; xiangyuezc@hotmail.com
}

Received 22 July 2021; Accepted 19 August 2021; Published 3 September 2021

Academic Editor: Gengxin Sun

Copyright ( 2021 Chao Zhang. This is an open access article distributed under the Creative Commons Attribution License, which permits unrestricted use, distribution, and reproduction in any medium, provided the original work is properly cited.

\begin{abstract}
Writing competence is crucial for second language learners. Studying strange attractors in the development of writing competence is essential in understanding the laws of language development of foreign students. This study is aimed at investigating the state and laws of the development of Chinese as a second language (CSL) writing competence. Mathematical modeling and phase space construction methods in sensor research were used to investigate strange attractors in high-level Chinese learners studying in China in the development of CSL from the perspective of complexity theory based on the measurement framework of complexity, accuracy, and fluency. The results showed the following: (1) there are trends in the concentration and volatility of trigonometric function in different dimensions; (2) the group dynamic characteristics of writing development in CSL are simulated precisely by mathematical modeling; and (3) there are strange attractors with lexical density in CSL writing development. The development of CSL writing tends to maintain the state of strange attractors. The strange attractor reflects regularity in the dynamic, complex, and chaotic development of Chinese for international students, revealing the probabilistic prediction competence of different states in the development of CSL.
\end{abstract}

\section{Introduction}

Quantitative experimental research under the paradigm of reductionism has explored the statistical law of language development by controlling changes of factors, making this approach the mainstream method of second language writing research since the 1980s. However, research through the lens of complexity theory has found that the development of learners' written language is a nonliner adaptive system, in which interconnectedness and dynamic changes produce significant individual characteristics. Therefore, language development is difficult to precisely predict. Without constructing the unified research paradigm, researchers have aimed to explore the consilience of reductionism and complexity theory to probe the statistical characteristics of second language development hidden in the differentiated developments of second language writing [1-3]. The attractor in complexity theory is considered a crucial construct that combines the two paradigms. An attractor is the state or pattern that complex systems tend to develop and may be the key to revealing different patterns of learners' lan- guage development and revealing the statistical law of language development in chaotic, dynamic individual differences $[4,5]$. This research is aimed at finding strange attractors in the development of Chinese as a second language (CSL) writing competence, with two novelties. (1) Few empirical studies have referred to attractors in second language development, which is still in the early stages of theoretical concept development [6-8]. The study of strange attractors in this research aids in further understanding this crucial construct in the complex system of language development; (2) there is a lack of effective methods to analyze attractors in the social sciences. The present study incorporates phase space construction and mathematical modeling methods used in sensor research to explore attractors. Previous studies have used a series of statistical methods, such as variance analysis, structural equation modeling, Monte Carlo simulation, and meta-analysis, which represent a challenge in exploring strange attractors. The application of interdisciplinary methods in the present research is the first instance of which academia has explored the strange attractors in the development of CSL writing competence, 
providing a reference for future research. Accordingly, this research uses the interdisciplinary methods of mathematical modeling and phase space construction that have been widely used in the sensor research to detect strange attractors in the development of CSL among high-level international students studying in China.

\section{Literature Review}

In this section, two paradigms in second language research are introduced, after which the definitions of attractors and strange attractors, as well as relevant research and recent developments, are reviewed.

\subsection{Theoretical Paradigms in Second Language Research.} Reductionism and complexity theory are two paradigms in research into second language writing development. Reductionism has been the mainstream method of second language writing research for decades, on which the development of second language writing has long been recognized as a rule-based process in which the effect of experimental variables on development can be explored by controlling variables through experimental design and using statistical methods based on mean values to examine the law of the average value of language development in statistics. Complexity theory is a theoretical cluster on which case studies have been conducted to produce a blueprint of second language development characterized by dynamic and individualized paths [7,9-12]. This field is challenging, and there is conflict between complexity theory and reductionism in perspectives and methods in that reductionism embraces the perspective of averaging individual differences in learners' language development.

The conflicting positions of the two paradigms hinder the unification of second language writing research in perspectives and methods. Therefore, researchers have called for the study of complex theory to concentrate on differences in language development and pay attention to statistical laws to explore language development patterns and the consilience between the two paradigms [2,13-15]. However, there are a few studies from a complexity theory perspective that have focused on statistical pattern characteristics that emerge from individual differences. For example, $\mathrm{Yu}$ and Lowie [8] found improvements in general developmental trends of both complexity and accuracy, whereas there is a complex interplay between complexity and accuracy that has transformed from a competitive relationship to a supportive relationship on a group level. However, the rules of learners' language development process in the relevant research have been inducted by concentrating on observation and speculation methods, lacking a mathematical discussion of the group's CSL development patterns.

2.2. Attractors and Strange Attractors. Attractors in complex theories are a crucial construct in addressing research gaps. Attractors are the state or behavior patterns of preference to which the complex system has a tendency to develop [5]. Phase space is a technique for analyzing attractors and is a prescribed space that represents all possible states of the system, also known as state space. The state of the system is defined by different dimensions. As such, a onedimensional phase space, for example, can be defined based on the writing scores of language learners, where each writing score of an international student corresponds to a unique point in the phase space. According to the characteristics of attractors in phase space, attractors can be divided into three types: fixed-point attractors, cyclic attractors, and strange attractors. The fixed-point attractor (Figure 1(a)) is a point in the phase space, which is a relatively constant state of the system in any dimension of the phase space. The cyclic attractor (Figure 1(b)) is multiple points in the phase space, representing the system in multiple states with periodic change. The strange attractor is the type of attractor and a feature of the complex system characterized with the most prominent chaos, also known as chaotic attractor. The butterfly-shaped area in Figure 1(c) is Lorenz's strange attractor [16]. The shape of the strange attractor is ambiguous, and its chaotic nature is reflected in the chaotic behavior of the system is disordered, but not random, and the trajectory has no obvious regularity [17]. Attractors are typical patterns emerging from system selforganization from the perspective of complexity theory, representing table and predictable aspects of development differences of the dynamic system, which are crucial ways in revealing different patterns of learner language development [4]. Studying strange attractors in the development of second language writing may reveal statistical rules contained in individual differences, which is a potential point of convergence to promote the unification of the two research paradigms.

Researchers have gained a limited understanding of attractors in the development of CSL in the field of applied linguistics. Complex theory originated in the field of natural sciences and has been widely applied in the field of sensor research, in which there exists proven methods for analyzing attractors in complex systems. The mathematical modeling method, for example, is an effective way of studying strange attractors by fitting development data captured by sensors. Through mathematical modeling, the state equation that characterizes the dynamic characteristics of the development of the CSL learners may be established, and the shape and boundary of the strange attractor in the phase space by simulating and drawing the state equation in the phase space can be analyzed. Therefore, using this interdisciplinary paradigm, this research takes international Chinese learners studying in China with high levels of language proficiency as the participants and examines strange attractors in the development of CSL writing, aiming to determine CSL development patterns hidden in individual differences based on complex theory.

\section{Method}

3.1. Research Questions. The present research focuses on patterns in the dynamic language development of high-level Chinese learners to examine potential strange attractors based on an analysis for characteristics of sensor data. 


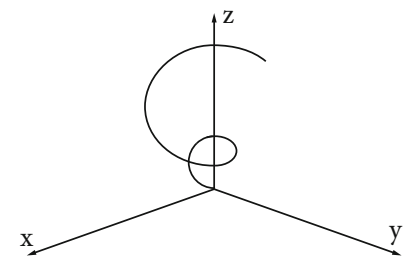

(a) Fixed-point attractor

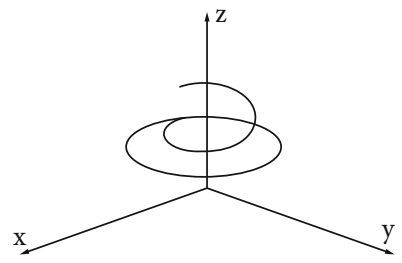

(b) Cyclic attractor

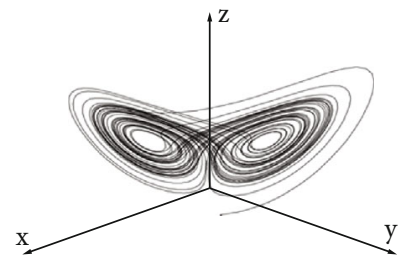

(c) Strange attractor

Figure 1: Three types of attractors.

Therefore, the following three research questions are formulated.

(RQ1) What are the statistical dynamic characteristics of the development of CSL writing for international students in China?

(RQ2) Can the statistical dynamic characteristics of the development of CSL writing by international students in China be fitted by mathematical models?

(RQ3) Are there any strange attractors in the development of CSL writing for international students in China? If so, what are their characteristics in the phase space?

3.2. Participants. This research focuses on a group of highlevel CSL learners' development of Chinese writing competence; 19 Chinese learners from four comprehensive universities in China were chosen as participants. Among these learners, 15 Thai international students studied in a first-class university in northern China, had passed the placement test, and were assigned to upper classes, all completing HSK Level 4. The other four international students were from two ordinary universities in the same province and had achieved HSK Level 5.

3.3. Writing Task. Sampling based on language teaching was used in this present research. In the research process, writing tasks were not assigned by the researchers; the writing texts produced by the learners within a limited time in class were collected. Participants were required to study teaching content ranging from six to eight units based on the writing curriculum. After each unit, there was a classroom writing task. The interval between the two tasks was approximately one month. The writing tasks were all based on selecting topics, involving learners' hometown, food, culture, personal career, daily life, school, critical thinking, etc. One-semester Chinese compositions written by international students were collected.

3.3.1. Writing Complexity. Complexity is a term that includes both lexical complexity and syntactic complexity. This research focuses on the lexical complexity of highlevel Chinese learners. Lexical complexity, which is also called lexical richness, contains three dimensions, namely, lexical density, lexical sophistication, and lexical diversity. In the present research, lexical density was chosen to measure the learners' lexical complexity. Lexical density refers to the ratio of the number of content words in the text to all words in the text [18]. Chinese content words include nouns, verbs, adjectives, adverbs, distinguishing words, numerals, quantifiers, pronouns, interjections, and ono- matopoeias. The formula for calculating the lexical density is as follows: lexical density = number of content words / number of words.

3.3.2. Writing Fluency. Writing fluency can be divided into process-based writing fluency and text-based writing fluency [19]. Process-based writing fluency is a time-related concept that mainly refers to the amount of text output per unit time, with a relatively single dimension. On the other hand, textbased writing fluency is related to text characteristics and is a multidimensional construct. Cluster analysis research shows that $\mathrm{T}$ unit length should be classified as complexity, and the number of words produced per minute is more appropriate for the concept of fluency, which represents the amount of text completed in a given time [20,21]. Based on the characteristics of this research, the following processbased measure was used to calculate writing fluency: writing fluency $=$ total number of symbols/total writing time.

When measuring total writing time used to calculate writing fluency, this research incorporated sensor technology. The sensor was able to accurately capture the writing time of the participants. When the pen tip touches the paper, the sensor under the paper will record the pressure-sensitive signal and start timing; when the pen tip leaves the paper, the sensor will stop timing. Through the sensor technology, the writing time of all participants was recorded.

3.3.3. Writing Accuracy. The accuracy of written CSL refers to the correct rate of words. Chinese composition is segmented into words, and the author and the writing teacher manually checked the words to measure accuracy. Word selection, collocation, and word meaning errors were all considered lexical errors. Lexical accuracy was measured by calculating the proportion of correctness in the composition text [22]. Since competence using letters and words belong to different constructs, they cannot be regarded as one dimension. As such, the present research did not consider letter errors when measuring lexical accuracy. The calculation method of accuracy is as follows: accuracy = number of errors/total number of symbols.

3.4. Phase Space. A tool for studying strange attractors is phase space in sensor research. After establishing the complexity, accuracy, and fluency index, Matlab was used to draw the three-dimensional phase space. A threedimensional coordinate system was constructed, in which the accuracy was the $x$-axis, fluency the $y$-axis, and lexical density the $z$-axis $(0<x<1, y>0, z>0)$. Any point in the 
three-dimensional coordinate system corresponded to only one state for language learners' Chinese writing accuracy, fluency, and complexity. Accordingly, the threedimensional coordinate system is the phase space of the CSL development. By constructing the phase space, the general scope of the development of complexity, accuracy, and fluency of international students' Chinese writing and the distribution characteristics of the development dynamics in each period in the phase space was observed. Based on this method, the strange attractors can be confirmed.

3.5. Data Analysis. The data analysis of this study was driven by the research questions. The first research question is aimed at examining the statistical characteristics of the development of Chinese writing text produced by learners. Therefore, the three indicators of complexity, accuracy, and fluency in the diachronic texts produced by studying abroad were calculated. The word segmentation software used the language technology platform developed by Harbin Institute of Technology [23], and the results of word segmentation and part-of-speech tagging were checked by the author of this study and a graduate student one by one to ensure that the word segmentation was correct. On the basis of this first stage, the number of content words, the number of errors, and the total number of characters were marked, and complexity, accuracy, and fluency were calculated one by one and recorded in a table in chronological order. An iterative graph of the development data of CSL writing competence was then constructed based on the task time as the abscissa and second language writing competence development indicator as the ordinate. The data of 21 participants were drawn into this coordinate system in turn. When fitting, coordinate time $t$ is replaced by the real-time data by a function. For example, if the first learner is sampled seven times and the second learner is sampled eight times, the number eight on the coordinate axis is replaced by number one. If the number of samples is set to time $t$, complexity, accuracy, and fluency are a function of task time. In addition, task time was marked on the abscissa; and complexity, accuracy, and fluency data were marked on the ordinate, by which a scatter distribution graph was drawn to visualize the value range of international students' writing complexity, accuracy, and fluency in the given tasks. The iterative graph represented participants' individual characteristics and micro changes in language development, whereas the scattered point distribution graph highlighted participants' statistical characteristics.

The second research question is aimed at fitting the statistical characteristics of the development of Chinese writing by international students through mathematics. In the field of natural science, the premise of examining a strange attractor involves constructing an equation of state [24]. Although the development of CSL writing of international students does not follow a specific equation in the social sciences, it is still possible to use mathematical models to fit statistical development dynamics [25]. By analyzing the development trend of CSL writing competence using scatter distribution, a mathematical model was constructed to simulate the development trend. This study used Matlab to fit the group development dynamics of the complexity, accuracy, and fluency for the development of CSL writing in China, on which a fitted state equation for the development of CSL writing was constructed based on the data of these three indicators. The change of the state with the equation in the phase space indicated a tendency for the development of CSL writing. According to nonlinearity view on the complex system, Matlab was used to calculate the root mean square error (RMSE) of the fitted equation to evaluate the degree of model fitting.

The third research question is aimed at examining the strange attractor based on the fitted equation of state. Therefore, data were imported into Matlab to draw the curve of the fitting equation development in the phase space. By simulating a large amount of data, the corresponding position of the equation in the phase space was visualized. The higher the simulation time, the clearer the tendency area of the equation, and the clearer the outline of the strange attractor in the phase space [17]. Similar to the method of establishing development dynamics by simulating a large amount of data, this technique was applied to the research based on dynamic systems theory; the Monte Carlo simulation is based on this idea [26]. In the present research, 800 time simulations were performed to confirm the characteristics of the strange attractor in the phase space.

\section{Results}

4.1. The Dynamics of the Development of CSL Writing for International Students in China. The iterative graph of the development data of CSL writing competence (Figure 2) presents significant individual differences among learners. The maximum value of accuracy is close to 1 , whereas the minimum value is close to 0.73 . The maximum value of writing fluency is close to 0.4 , and the minimum value is close to 0.05 . The maximum value of lexical density is above 0.9 , and the minimum is close to 0.6. In addition, no two segments of the same curve can be found in the graph, indicating that the development path of the Chinese language of international students is unique. The development of the second language writing is different, showing progress, retrogression, variability, and turbulence.

To visualize the development of CSL writing competence, a scatter plot of complexity, accuracy, and fluency data was drawn (Figure 3), in which accuracy and lexical density overlap and overall line changes are relatively stable. The lexical density (gray area), for example, presents fluctuates of maxima and minima over time, that is, from the first to the eighth writing index changes are approximated by a trigonometric function (a wavy line, closer to a sine function). In the change of lexical density, the index value reaches a lower maxima at the second time and reaches a higher maxima at the third time, dropping slightly and then rising. The overall range of changes is clear, in which the lowest value is approximately 0.6 , and the highest value is 0.979 .

The two-dimensional diagram provides a description of the individual characteristic and group development of learners' CSL writing competence, clarifying the direction 


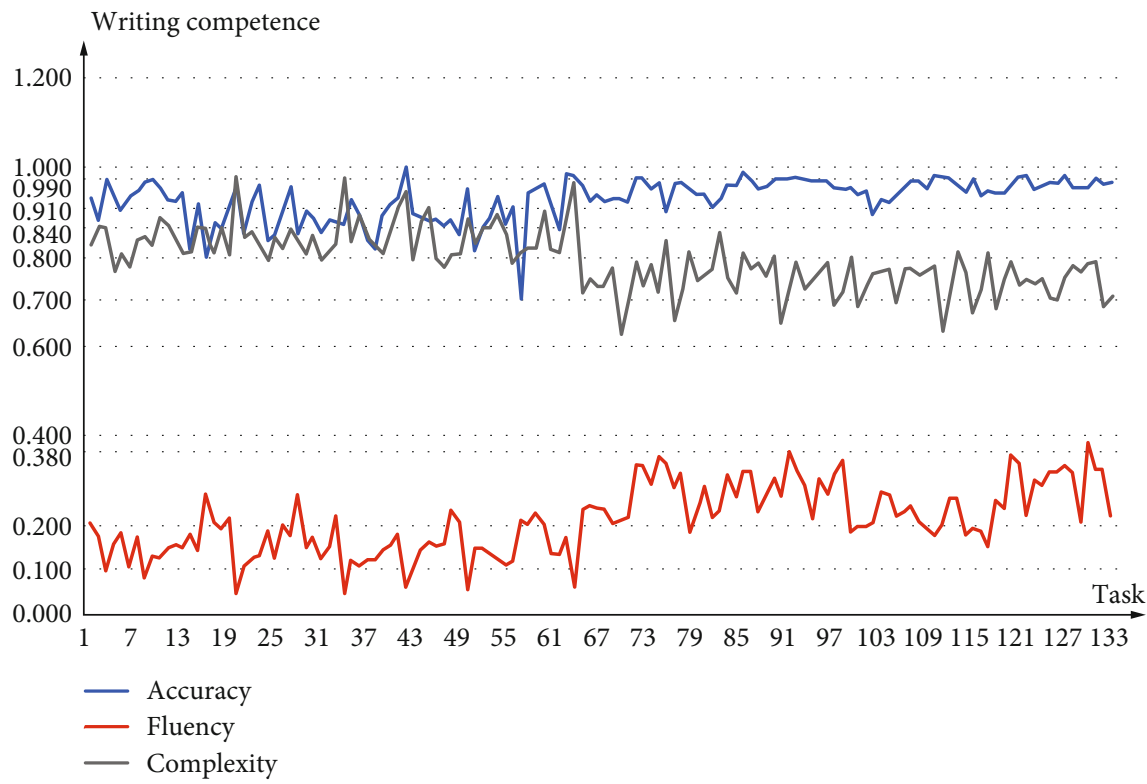

FIGURE 2: Iterative graph of the development data of CSL writing competence.

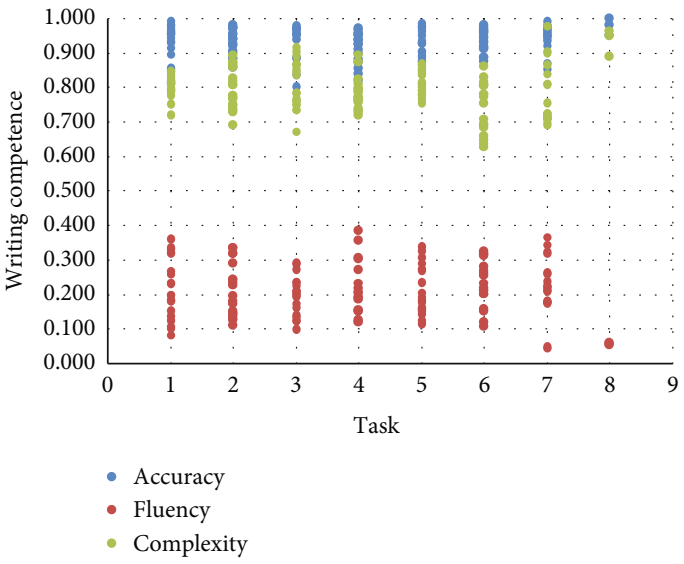

Figure 3: Scattered distribution map of the development data of CSL writing competence.

for examining strange attractors for the current research. There may be individual differences with fluctuations and variabilities in language competence development, showing stable characteristics in statistics and indicating that the statistical law exists. In addition, the fluctuation of the index shows the characteristics of trigonometric functions, and the development process is nonlinear, showing that it is reasonable for trigonometric function models (e.g., Fourier transform) to construct a mathematical model for the development tendencies of Chinese writing competence. Therefore, in the present study, sine and cosine functions were chosen to construct a mathematical model to fit learners' group development dynamics.

4.2. Mathematical Model of the Development of CSL Writing Competence. Mathematical modeling describes the dynamic trend of the development of international students' CSL writing competence. In the present study, function fitting was used with the task time as an independent variable, with complexity, accuracy, and fluency as dependent variables. After several adjustments, the fitting function of CSL writing competence was established (Table 1). After calculating accuracy $(\mathrm{RMSE}=0.003)$, fluency $(\mathrm{RMSE}=0.013)$, and complexity $(\mathrm{RMSE}=0.034)$, the root mean square error of the fitting function was lower than 0.1 , showing that the development of the three dimensions of complexity, accuracy, and fluency was effectively fitted for international students' CSL writing competence.

The graph of the fitting function in the abscissa $[1,5]$ interval is presented in Figure 4, which is fitted through discrete points; that is, there are only integer time of tasks on the abscissa, and the fitting data between two adjacent integer points only has mathematical meaning. The graph of the accuracy fitting function (Figure 4(a)) shows that the participants' CSL writing accuracy is approximately 0.95 in the first task, slightly increases in the second task, and continues to decline from the third to the fifth task. The graph of the fluency fitting function (Figure 4(b)) reflects the tendency of the participants' fluency in CSL writing from the first task to the third task and then continues to rise. The graph of the complexity fitting function (Figure 4(c)) shows an overall trend of the participants' CSL writing complexity falling and rising. The function graphs accurately present the statistical characteristics of the development of complexity, accuracy, and fluency in the scatter distribution map of the development data of CSL writing competence.

4.3. Strange Attractors in the Development of CSL Writing. The attractor is a tendency pattern for the development of complex systems and is mapped as a region in the phase space. Therefore, the phase space graph was constructed to examine a strange attractor with accuracy as the $x$-axis, fluency as the $y$-axis, and vocabulary density as the $z$-axis. The three-dimensional scatter graph of the state equation of 
TABLE 1: Fitting function and evaluation of fitting degree of CSL writing development.

\begin{tabular}{lcc}
\hline Dimension & RMSE & Function \\
\hline Accuracy & 0.003 & Accuracy $=0.917+0.075 \sin (3.272 t)$ \\
Fluency & 0.013 & Fluency $=0.24+0.137 \cos (t)$ \\
Complexity & 0.034 & Density $=0.8+0.11 \sin (0.14 t) \sin \left(0.052 t+t^{2}\right)$ \\
\hline
\end{tabular}
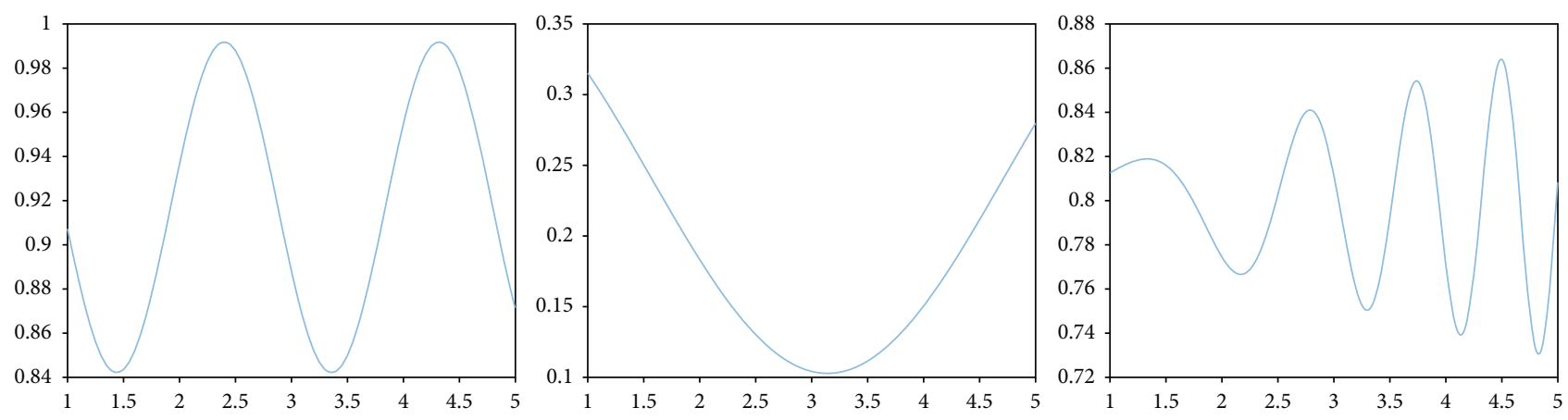

FIgURE 4: The fitting function graph of CSL writing accuracy, fluency, and complexity.

complexity, accuracy, and fluency development (Figure 5) shows that the scatter points are mainly blue and blue-green, indicating that the lexical density is distributed in interval $[0.6,0.9]$, demonstrating the possibility of the attractors and the tendency of the complex system of international students in CSL writing.

According to the analyses in Section 4.2, the fitted equation of state for strange attractor is as follows:

$$
\left\{\begin{array}{l}
x=0.917+0.075 \sin (3.272 t) ; \\
y=0.24+0.137 \cos (t) ; \\
z=0.8+0.11 \sin (0.14 t) \sin \left(0.052 t+t^{2}\right) .
\end{array}\right.
$$

Based on the fitted equation of state, the function of the strange attractor simulated 800 times in the interval $[1,50]$ was drawn as three-dimensional graph (Figure 6), demonstrating that the data points are distributed in the interval of lexical density $[0.70,0.91]$, accuracy [0.84, 0.99], and fluency $[0.10,0.38]$ to form a rectangular area approximately, in which a large number of data points are distributed in the graph of fitted function, and a few data points outside the rectangular area will return to the area soon, indicating the tendency of development of CSL writing competence for international students. The behavior of the system in this phase space region is disorderly and unpredictable, though its boundary is relatively clear. Therefore, the rectangular area in the phase space is confirmed to be a strange attractor. Comparing Figures 2 and 3, the strange attractor is in the scatter of the development of CSL writing competence, and the fitting equation is consistent with development distribution.

By examining the language ability development data of 19 participants, the accuracy of their CSL writing development left strange attractors 10 times, accounting for $7.5 \%$ of total times. Furthermore, participants' fluency development left strange attractors eight times, accounting for $6.0 \%$ of total times, the complexity development left strange attractors 16 times, accounting for $12.0 \%$ of total times. The development of the second language writing of 17 participants escaped from the strange attractor, whereas the development of the second language writing of only two participants (Lin and Chen) escaped from the attractor for two consecutive tasks (the value of accuracy is 37 and 38 , and the value of lexical density is 70,71$)$. The Chinese writing development data of two international students (Table 2) shows that Lin's writing accuracy was lower than the minimum (i.e., 0.84) of strange attractor twice in the third and fourth tasks, whereas Chen's writing complexity was lower than the minimum (i.e., 0.70) of strange attractor in the last two tasks. Once the second language writing development of other learners escaped from the rectangular area, they had to return to the strange attractor state in the next writing task. The above analysis shows that the development of Chinese writing by international students tends to develop towards and remain in strange attractors.

\section{Discussion}

A statistical analysis of the three indicator dimensions of the development of the CSL writing competence of international students was conducted to examine the statistical characteristics of the development of Chinese writing competence among international students and the strange attractors in the phase space. Through the development curve fitting and mathematical modeling, the strange attractor in the development of CSL writing competence was confirmed. These findings show difference, regularity, predictability, and complex are interconnected in language development. These features are discussed in the following section. 


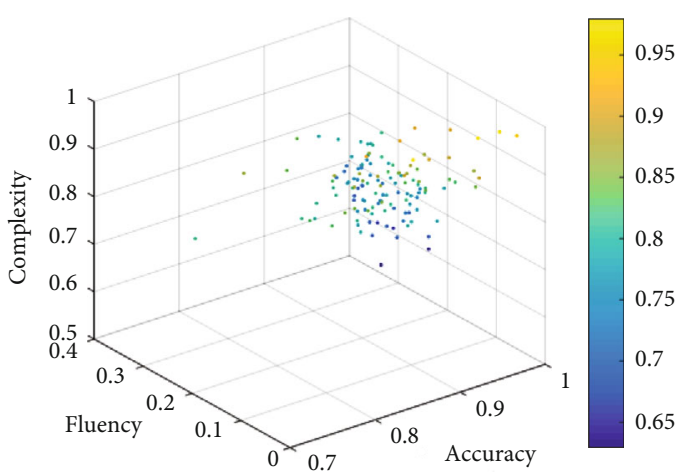

FIGURE 5: Scatter graph of the state equation of complexity, accuracy, and fluency development.

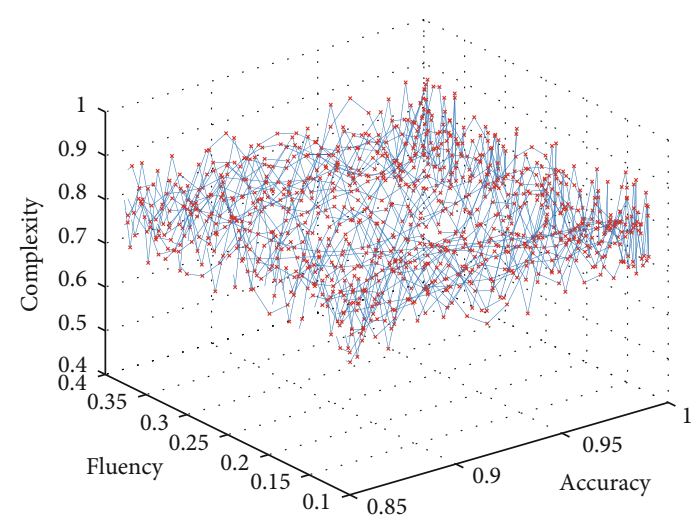

FIgURE 6: Strange attractor.

5.1. The Chaos of Strange Attractors in the Development of CSL. The results on the development dynamics of CSL writing competence show that there are a large number of individual differences in language development manifested in many aspects of development dynamics. There are no two participants with the same development dynamics, and the development curve of the learners' CSL writing competence shows increases, decreases, leaps, and temporary stagnation. The iterative graph of the complexity, accuracy, and fluency of the CSL writing competence shows that the development curve of international students in different dimensions is the identity of each participant. Therefore, learners' development dynamics in each dimension are unique, and the chaotic development trajectory of the strange attractor reflects this characteristic. Complexity theory provides a view that the environment as a complex system constantly changes, in which different learners have different system components in a period of time (for example, a month). Therefore, there are individual differences in the interconnectedness of the subsystems, and it is the dynamic interaction of the system elements that forms the dynamics of differentiated language development [27]. Interconnectedness, the prominent feature of the complex system, dominates the interactions of the subsystems of complexity, accuracy, and fluency in the development of CSL writing to form individual characteristics, in which learners' language development shows chaotic characteristics, making it almost impossible to precisely pre- dict the development path of learners' language competence [13]. Due to the variability, interconnectedness, and sensitivity to the initial state of the system, the subsystems in a complex system are in constant change. As a consequence, learners will never follow the same path of second language writing development $[11,26]$.

5.2. Statistical Law of Strange Attractors in the Development of CSL Writing Competence. Strange attraction is a model of development of foreign students' Chinese in the dynamics of individual differences and chaos. The development of Chinese writing ability of foreign students in singular attractor changes dynamically throughout the process but tends to remain within a specific range with vocabulary density $[0.70,0.91]$, accuracy $[0.84,1]$, and fluency $[0.10,0.38]$ in a specific state area. The results of the lexical density dimension are consistent with previous research results on highlevel CSL writing [28], indicating that the strange attractor found in this study may be a common state of the development of high-level Chinese in China. The dynamic development of learners' writing competence presents a tendency towards strange attractors, in which the development of Chinese writing for international students escapes for a short time but then returns (Figure 1). The graph of the equation of state (Figure 5) also displayed this feature of the strange attractors, indicating that the strange attractor is a state in which participants' Chinese writing competence tends to stay. In addition, the participants are CSL learners with high-level proficiency. Therefore, it may be considered that the strange attractor is a state achieved by the participant group through self-organization of the complex system of Chinese development after long-term acquisition; the strange attractor is also a state in which learners tend to develop. Strange attractors seem to "attract" the development of Chinese writing by international students in China from beginner to a high-level proficiency Chinese learners, which is the statistical law and certainty that emerges from the interaction and chaotic development of CSL writing competence.

The analysis of strange attractors in the development of Chinese writing competence by international students suggests that construction emergence may play a key role in this process. Construction is the pairing of form and semantics/function with conventional characteristics [29]. Emergence is the showing of new states of the system that cannot be explained with existing parts and interactions [30]. Lexical density is the proportion of content words in a text, and using construction may help language learners to call on more language resources and organize more complex sentences, thus, significantly increasing their lexical density and generating more fluent language [31, 32]. Although the participants were Chinese learners with high-level proficiency, the analysis showed that some of the more advanced constructs (e.g., Chinese slot and frame construction) were in constant change, in which the degree of solidification of the construction representation was not high, and the constructs were still in the process of acquisition. The development system of CSL writing competence in the strange attractor is not stable; there are mismatches and 
TABle 2: Data on Chinese writing development of two international students in China.

\begin{tabular}{|c|c|c|c|c|c|c|c|c|c|}
\hline & No. & Accuracy & Fluency & Complexity & & No. & Accuracy & Fluency & Complexity \\
\hline \multirow{7}{*}{ Lin } & 1 & 0.930 & 0.123 & 0.837 & \multirow{7}{*}{ Chen } & 1 & 0.957 & 0.235 & 0.719 \\
\hline & 2 & 0.899 & 0.112 & 0.893 & & 2 & 0.926 & 0.244 & 0.750 \\
\hline & 3 & 0.835 & 0.123 & 0.846 & & 3 & 0.941 & 0.239 & 0.736 \\
\hline & 4 & 0.819 & 0.122 & 0.828 & & 4 & 0.923 & 0.235 & 0.736 \\
\hline & 5 & 0.894 & 0.143 & 0.804 & & 5 & 0.932 & 0.207 & 0.778 \\
\hline & 6 & 0.919 & 0.158 & 0.861 & & 6 & 0.930 & 0.213 & 0.629 \\
\hline & 7 & 0.933 & 0.181 & 0.906 & & 7 & 0.922 & 0.218 & 0.693 \\
\hline
\end{tabular}

intensification in the process of construction emergence, which evolves on an individual level and shows as statistical characteristics. In addition, there is a feedback mechanism in the process of learners' interactions with the environment. Feedback refers to the impact of the current state on the subsequent state of the system, and the effect of the negative feedback is one of the most common attractor influence system mechanisms [33]. In this study, once the development path of CSL writing competence of international students in China leaves the strange attractor, there seems to be an invisible force that urges the development state to return to the singular attractor. This process derives from the system's negative feedback mechanism. The strange attractor of the learner's language complexity, accuracy, and fluency is the result of the long-term self-organization and stability of the system, in which the attractor is adapted to the cognitive resources that the learner is able to call. When the need of a certain dimension exceeds the learner's cognitive competence at a certain level (e.g., a learner tries to use an unfamiliar construction), the three dimensions of language performance compete for cognitive resources. A competitive growth forms in the internal dimensions, further decreasing available cognitive resources $[34,35]$. The completive state is a negative experience for learners and cannot be maintained for an extended period, which triggers the negative feedback mechanism of the system and reduces the difference between the states of the complex system and the strange attractors, showing that the system tends to return to the strange attractor after leaving the strange attractor.

There are similarities in the development path of Chinese writing competence among learners with the same strange attractors, and the system shows order under these strange attractors. The self-organization of the system leads to the order of the complex system of CSL writing competence to reduce the degree of freedom of the system [36] and finally converge the development of the system to several possible patterns. Variability and self-organization are crucial characteristics of complex systems, in which variability leads to individual differences and unpredictability in the development of learners' CSL writing competence, whereas self-organization makes the development of learners' CSL writing more statistical to eventually form attractors. A stable system makes the macroscopic characteristics of nonlinear systems more prominent [33]. Strange attractors represent the tendency of learners' writing competence to develop in the form of accuracy, fluency, and complexity. The strange attractor, for example, corresponds to learners' tendency towards accuracy $[0.84,1]$, fluency $[0.10,0.38]$, and lexical density $[0.70,0.91]$ in the three-dimensional phase space region. The fitting function of the development state and the superimposed graph of writing competence development of all indicators show that the development of the group at a specific time presents a specific interval characteristic.

5.3. Probabilistic Predictability of Strange Attractors in the Development of CSL Writing Competence. The essence of science is predictability ([17] p.6). In the past, language research based on complexity theory has avoided mentioning the "predictability" of language, and the interconnectedness and openness of language development complex system make it almost impossible to precisely predict language development as a chaotic trajectory inside the strange attractor. Therefore, empirical research based on complex theory has used the "retrospective" approach in essence, concentrating on the reality of language development. The research source must be the dynamic trajectory of the system that has occurred [13]. The strange attractor indicates that, when the individual differences and laws of language development are considered, language development shows significant statistical laws that can be predicted to a certain extent. The characteristics of strange attractors in the development of CSL writing competence indicate that it may predict the dynamic range of language development in the next moment although it is difficult to precisely predict the development of the next moment and find clear rules from the dynamics, which is the consilience between individual differences and statistical rules, as well as reductionism and complexity in the development of a second language.

The development of learners' CSL writing competence tends to be in state with accuracy in $[0.84,1]$, fluency in $[0.10,0.38]$, and complexity in $[0.70,0.91]$, in which the accuracy of the second language writing of all participants in the state last for at least six months. The probability of the participant maintaining the strange attractor in the seventh month is higher than the probability of phase transition to other states. For individuals such as Chen, the probability that the lexical density of the next written text as [0.629, 0.778] is significantly higher than the probability of leaving this interval, in that the lexical density of her writing in the first six months is at the strange attractor. The language performance in the first six months is a condition, and the conditional probability of the state at time $t$ may be expressed as $P\left(X_{t} \mid X_{t-1}, X_{t-2}, \cdots, X_{1}\right)$, showing that it is possible to 
predict that the same attractor state will be maintained at a later time and predict the development interval for attractors. This prediction is reasonable based on the characteristics of the attractor based on previous sufficient observations. In other words, the language development state of the learner in the attractor at the next moment is not infinite, which conforms to the conditional probability distribution that the probability of being in a different state at the next moment is different, which may be predicted. The analysis shows that language development can be predicted to a certain extent, and this prediction conforms to the probability distribution. The extent to which language development can be predicted is determined by the state of the strange attractor, which depends on the conditional probability corresponding to the development of the learner in the dimension. The state of attractor, which is difficult to measure directly, is called the hidden state. The state of complexity, accuracy, and fluency, which can be measured directly, is the explicit state. The hidden state implies the complexity of the laws of language; it is difficult to find the laws from a bunch of individual data, and the hidden state must be found using statistical tools. However, the attractor, as a hidden state, provides the possibility of predicting the dynamic path of language development.

\section{Conclusion}

Based on an analysis of the characteristics of attractors, this study demonstrated the coexistence of individual differences and statistical laws in the development of CSL writing. Individual difference was manifested in the unique dynamic curve of language development, and statistical law was manifested learners' CSL writing development, which preferred certain intervals with fluctuations. In this study, the state equation for the development of CSL writing competence was constructed, and characteristics of participants' CSL writing competence development were simulated using mathematical modeling and phase space construction. The trajectory of the development of CSL writing in the phase space based on the equation of state suggests that there is a strange attractor in the development of CSL writing competence among international students in China, which are mapped in the phase space as accuracy in $[0.84,1]$, fluency in $[0.10,0.38]$, and complexity in $[0.70,0.91]$. The strange attractor shows a more general, tendentious state in CSL dynamic development with significant individual differences, reflecting the statistical law of the development of the CSL and the probabilistic predictive characteristics of the state.

The theoretical significance of this research is to use the strange attractor as an approach to address key issues of complexity theory and reductionism, or to what extent is language development individual and to what extent do laws or patterns exist [2]. The emergence of complexity theory interrupts the authority of reductionism. However, there is a fundamental difference between the two paradigms on language development. The results indicate the consilience of complexity theory and reductionism in language research, and the research paradigm may be further expanded. In addition, this research has value for the teaching and research of second language writing. First, reductionist research can use diachronic experimental design to explore the dynamics and complexity of potential factors and confirm which types of learners are more in line with statistical law under reductionism. Research based on complexity theory may use a large sample, focusing on statistical law and patterning characteristics contained in the dynamics of individual differences. Second, as a hidden state, the strange attractor may become a classification label for learners to guide the implementation of differentiated teaching. This research used interdisciplinary paradigms such as mathematical modeling of dynamics in statistics, providing an effective method to detect strange attractors. Third, the strange attractor found was a law and phenomenon. The detection of attractors in learners' second language development helps language teachers to determine teaching strategies and analyze control parameters. Teachers could assist learners in maintaining a positive state and escaping from the negative aspects of CSL development. Fourth, using sensors to detect participants' writing time and sensor-based mathematical modeling methods to detect strange attractors in this study may provide references for language research.

The method of detecting the strange attractor in this study could be further improved. The study on attractors in the language development is still in its early stages, and the method of detecting attractors in language development is still in its exploratory stage. Therefore, this research used mathematical modeling, spatial mapping, and other sensor fields to establish strange attractors. These interdisciplinary methods still have limitations in the field of language research. The individual learner's development is complex and chaotic, making it impossible to accurately follow a certain mathematical equation. The fitted state equation is the mathematical simulation of the dynamic tendency of CSL learners' development. Although it may often be necessary to eliminate some details in language research to explore statistical laws, more accurate models need to be further explored to more precisely describe the actual state of language development.

\section{Future Research}

The limitations of this research may be addressed in future research. The method of probing strange attractors should be enhanced in said research. Because academic research into attractors in language development of learners is still in its early stages, the method of confirming attractors in language development is also still in its exploratory stage. In the future, more interdisciplinary methods may be incorporated to explore strange attractors in further detail (e.g., computer modeling based on deep learning). Furthermore, computer modeling can address limitations that are difficult to solve with mathematical modeling and more accurately depict the development of CSL writing competence among foreign students in China. In addition, a further study of a longer duration may be conducted to describe the attractor formation process and learners' language development dynamics more clearly, comprehensively describing the 
distribution of attractors among language learners and the group law of attractors. Furthermore, future research may use a higher number of participants to more comprehensively reveal the distribution law and formation process of attractors.

\section{Data Availability}

The data used to support the findings of this study are included within the article.

\section{Conflicts of Interest}

The authors declare that they have no conflicts of interest.

\section{Acknowledgments}

The author is grateful for the comments of his Ph.D. advisor.

\section{References}

[1] L. M. Kallemeyn, J. N. Hall, and E. Gates, "Exploring the relevance of complexity theory for mixed methods research," Journal of Mixed Methods Research, vol. 14, no. 3, pp. 288-304, 2020.

[2] D. Larsen-Freeman, "Complexity theory," in The Routledge Handbook of Second Language Acquisition, S. M. Gass and A. Mackey, Eds., Routledge, London, New York, 2012.

[3] C. Zhang and R. Ma, "The effect of textual glosses on L2 vocabulary acquisition: a meta-analysis," Language Teaching Research, vol. 5, 2021.

[4] P. Hiver, "Attractor states," in Motivational Dynamics in Language Learning, Z. Dörnyei and P. D. MacIntyre, Eds., pp. 2028, Multilingual Matters, UK, 2014.

[5] E. Thelen and L. B. Smith, A Dynamic Systems Approach to the Development of Cognition and Action, The MIT Press, Cambridge, MA, 1994.

[6] C. M. Amerstorfer, "The dynamism of strategic learning: complexity theory in strategic L2 development," Studies in Second Language Learning and Teaching, vol. 10, no. 1, pp. 21-44, 2020.

[7] G. G. Fogal, "Investigating variability in L2 development: extending a complexity theory perspective on L2 writing studies and authorial voice," Applied Linguistics, vol. 41, no. 4, pp. 575-600, 2020.

[8] H. Yu and W. Lowie, "Dynamic paths of complexity and accuracy in second language speech: a longitudinal case study of Chinese learners," Applied Linguistics, vol. 41, no. 6, pp. 855877, 2020.

[9] K. Baba and R. Nitta, "Phase transitions in development of writing fluency from a complex dynamic systems perspective," Language Learning, vol. 64, no. 1, pp. 1-35, 2014.

[10] D. Larsen-Freeman, "On language learner agency: a complex dynamic systems theory perspective," The modern language journal, vol. 103, supplement 2016, pp. 61-79, 2019.

[11] D. Larsen-Freeman and L. Cameron, Complex System and Applied Linguistics, Oxford Univ. Press, New York, 2008.

[12] W. M. Lowie and M. H. Verspoor, "Individual differences and the ergodicity problem," Language Learning, vol. 69, Supplement 1, pp. 184-206, 2019.
[13] Z. Dörnyei, "Researching complex dynamic systems: 'retrodictive qualitative modelling' in the language classroom," Language Teaching, vol. 47, no. 1, pp. 80-91, 2014.

[14] T. Wrigley, "The problem of reductionism in educational theory: complexity, causality, values," Power and Education, vol. 11, no. 2, pp. 145-162, 2019.

[15] B. Yuan and Y. Lin, "Directionality and complexity of L1 transfer in L2 acquisition: evidence from L2 Chinese discourse," International Review of Applied Linguistics in Language Teaching, vol. 57, no. 4, pp. 377-416, 2019.

[16] E. N. Lorenz, "Deterministic nonperiodic flow," Journal of the Atmospheric Sciences, vol. 20, no. 2, pp. 130-141, 1963.

[17] J. C. Sprott, Strange Attractors: Creating Patterns in chaos, M \& T Books, New York, 1993.

[18] J. Ure, "Lexical density: a computational technique and some findings," in Talking about Text, Birmingham: English Language Research, M. Coultard, Ed., pp. 27-48, University of Birmingham, 1971.

[19] M. M. A. Latif, "What do we mean by writing fluency and how can it be validly measured?," Applied Linguistics, vol. 34, no. 1, pp. 99-105, 2013.

[20] N. A. Chenoweth and J. R. Hayes, "Fluency in writing," Written Communication, vol. 18, no. 1, pp. 80-98, 2001.

[21] K. Wolfe-Quintero, S. Inagaki, and H.-Y. Kim, Second Language Development in Writing: Measures of Fluency, Accuracy, \& Complexity, Second Language Teaching \& Curriculum Center, Hawaii, 1998.

[22] J. Read, Assessing Vocabulary, Cambridge University Press, Cambridge, 2000.

[23] W. Che, Z. Li, and T. Liu, "LTP: a Chinese language technology platform," in proceedings of the Coling 2010: demonstrations, Beijing, China, 2010.

[24] R. A. Meyers, Ed., Encyclopedia of Complexity and Systems Science. Springer Reference, Springer, New York, London, 2009.

[25] S. C. Chapra, Applied Numerical Methods with MATLAB for Engineers and Scientists, McGraw-Hill Education, New York NY, Fourth edition edition, 2018.

[26] M. H. Verspoor, K. deBot, and W. Lowie, A Dynamic Approach to Second Language Development: Methods and Techniques, John Benjamins, Amsterdam, 2011.

[27] D. Larsen-Freeman, "Complexity theory: the lessons continue," in Complexity Theory and Language Development: In Celebration of Diane Larsen-Freeman, L. Ortega and Z. H. Han, Eds., pp. 11-50, John Benjamins, Amsterdam/Philadelphia, 2017.

[28] C. Huang and N. Xue, "Digital language resources and NLP tools," in The Routledge Handbook of Chinese Applied Linguistics, C.-R. Huang, J.-S. Zhuo, and B. Meisterernst, Eds., pp. 483-497, Routledge, London, 2019.

[29] N. C. Ellis, "Constructions, chunking, and connectionism: the emergence of second language structure," in Blackwell handbooks in linguistics, The handbook of second language acquisition, C. Doughty and M. H. Long, Eds., Blackwell, Malden, MA, Oxford, 2003.

[30] J. H. Holland, "Emergence: from chaos to order (3. Paperback print)," in Helix books, Perseus books, Cambridge, Mass, 1998.

[31] N. C. Ellis, "Language acquisition as rational contingency learning," Applied Linguistics, vol. 27, no. 1, pp. 1-24, 2006.

[32] A. E. Goldberg, Constructions: A Construction Grammar Approach to Argument Structure Originally Presented as the 
Author's [PhD Thesis], University of California, Chicago, London, 1992.

[33] W. Banzhaf, "Self-organizing systems," in Springer Reference. Encyclopedia of Complexity and Systems Science, R. A. Meyers, Ed., pp. 8040-8050, Springer, New York, London, 2009.

[34] P. Skehan, A Cognitive Approach to Language Learning, Oxford University Press, Oxford, UK, 1998.

[35] P. Van Geert, "A dynamic system model of cognitive and language growth," Psychological Review, vol. 98, no. 1, pp. 3-53, 1991.

[36] T. DeWolf and T. Holvoet, Eds., Emergence Versus Self-Organisation: Different Concepts but Promising when Combined, vol. 1 of Engineering Self-Organising Systems: Methodologies and Applications, Springer, Berlin, 2005. 